\title{
Broadband cavity-enhanced absorption spectroscopy for real time, in situ spectral analysis of microfluidic droplets
}

\author{
Simon R.T. Neil, ${ }^{a}$ Cathy M. Rushworth,${ }^{b}$ Claire Vallance, ${ }^{b *}$ and Stuart R. Mackenzie ${ }^{a *}$ \\ Received (in XXX, XXX) Xth XXXXXXXXX 20XX, Accepted Xth XXXXXXXXX 20XX \\ DOI: 10.1039/b000000x
}

Broadband cavity enhanced absorption spectroscopy has been used to record, in real time, the absorption spectrum of microliter volume aqueous phase droplets within a microfluidic chip assembly. Using supercontinuum radiation 10 and broadband coated external mirrors, the full visible spectrum $(430<\lambda / \mathbf{n m}<700)$ of each passing droplet is acquired in situ at high repetition rates $(273 \mathrm{~Hz} / 3.66 \mathrm{~ms}$ acquisition time) and high sensitivity $\left(\alpha_{\min }<10^{-2} \mathrm{~cm}^{-1}\right)$. The possibilities for further improvements in sensitivity and 15 acquisition rate using custom designed chips are discussed.

A wide variety of optical detection methods have been applied to microfluidic systems, including fluorescence, absorbance, chemiluminescence, surface plasmon resonance, and Raman spectroscopy. ${ }^{1}$ In the analysis of macroscopic chemical samples, 20 direct absorbance spectroscopy is considered a universally applicable analytical technique. Its application to microfluidic systems, however, presents significant challenges due to the dramatically reduced optical pathlengths involved. Liquid-core waveguides and fiber optics have been employed to guide light 25 through microchannels in order to increase the optical pathlength sampled. ${ }^{2,3}$ However, whilst waveguide-based approaches are well suited to integration within microfluidic devices, paving the way for commercially viable "on-chip" techniques in which the light source, fluidic elements and detector are all incorporated 30 within a single monolithic platform, ${ }^{1,4,5}$ they remain relatively insensitive compared with other on-chip optical detection methods. Furthermore, the integration of waveguides places constraints on the chip and channel geometry and can introduce significant light losses during coupling and detection.

35 One convenient way to increase the effective path-length is to use multiple pass techniques. A simple way to achieve this is to place the sample within a high finesse optical cavity such as that formed between two highly reflective mirrors. Cavity-enhanced spectroscopic techniques of this type are well established in gas-

${ }_{40}$ phase absorbance measurements ${ }^{6}$ and are becoming increasingly popular for liquid phase studies. ${ }^{7,8}$

Integration of multiple-reflection mirrors into microfluidic chips has been achieved previously using coated capillaries ${ }^{9}$ or, for protein / bulk solution detection with integrated gold mirrors ${ }^{10}$ 45 although difficulties in alignment led to modest gains in sensitivity compared with single pass measurements.

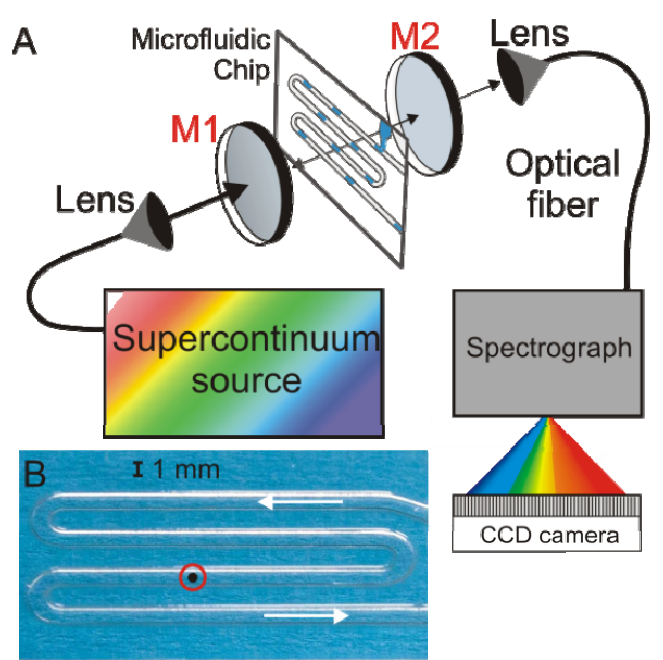

Fig. 1 (A) Experimental BBCEAS setup. (B) The microfluidic chip and channels (1 $\mathrm{mm}$ in width and $320 \mu \mathrm{m}$ in depth), as 50 viewed along the optical axis of the cavity. The position and estimated size of the optical beam is shown as a black dot.

Here we describe the first application of truly broadband cavityenhanced absorption spectroscopy (BBCEAS) to in situ analyte detection within microfluidic droplets. In BBCEAS, broadband ${ }_{55}$ light is continually injected into an optical cavity formed between two broadband, highly reflective mirrors. The time-integrated light intensity emerging from the cavity, $I(\lambda)$, is dispersed into its constituent wavelengths before detection. For small intracavity light losses and high mirror reflectivities, the wavelength${ }_{60}$ dependent (decadic) absorbance $A=\varepsilon C l$, may be approximated from measurements made in the presence, $I$, and absence, $I_{0}$, of a sample, as

$$
2.3026 A=2.3026 \varepsilon C l=\frac{\left(I_{0} / I\right)-1}{\mathrm{CEF}},
$$

where $\varepsilon$ is the molar absorbtivity, $C$ the absorber concentration ${ }_{65}$ and $l$ the single-pass optical pathlength through the sample. ${ }^{11}$ $\mathrm{CEF}$ is the cavity-enhancement factor, defined as the ratio of the measured absorbance to that for a single pass.

The experimental setup used here is shown schematically in Fig. 1(A). Two highly reflective mirrors, M1 and M2, (Layertec, ${ }_{70} \mathrm{R}_{400 \ldots . .800 \mathrm{~nm}}>0.9985,1 \mathrm{~m}$ radius of curvature) form a stable linear cavity of length $12 \mathrm{~cm}$. A microfluidic chip (Fig $1 \mathrm{~B}$ ) is inserted into the optical cavity at normal incidence to the optical beam 
such that the light within the cavity passes through a single microfluidic channel.

The microfluidic chip used in these experiments was fabricated according to Section 3 of Ref 12. Channels of width $1 \mathrm{~mm}$ and 5 depth $320 \mu \mathrm{m}$ were fabricated in UV-curing optical adhesive (Norland NOA81), sandwiched between two $\sim 140 \mu \mathrm{m}$ thick microscope cover slides. Liquid flows into and out of the chip through Nanoports (Upchurch Scientific). Dual inlets allow for measurements to be made in continuous- or droplet-flow regimes.

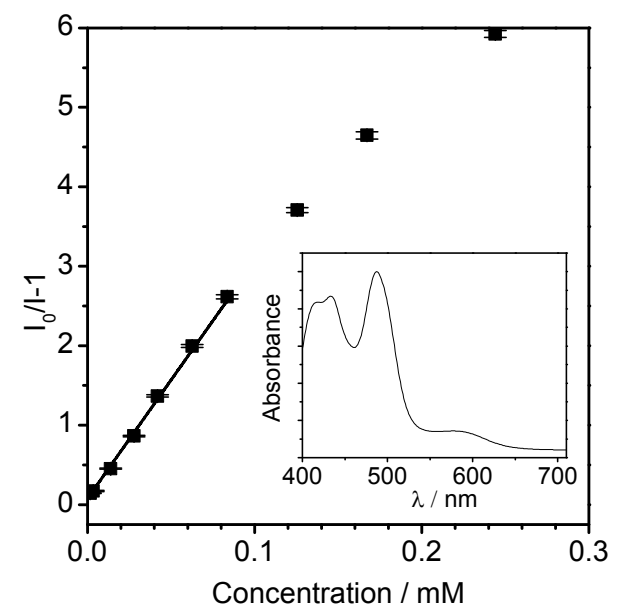

Fig. $2 \mathrm{I}_{0} / \mathrm{I}-1$ (at $487 \mathrm{~nm}$ ) versus $\mathrm{Ir}(\mathrm{IV})$ concentration. Data points represent the average of 15 measurements, the standard deviation of which is shown. The solid line represents an error-weighted regression $\left(\mathrm{R}^{2}=0.996\right)$ through the linear region of the graph.

Inset, normalized absorption spectrum of $\operatorname{Ir}(\mathrm{IV})$.

In order to record broadband spectra at high repetition rates a spectrally brilliant commercial supercontinuum (SC) source (NKT, Koheras SuperK Power Blue) in employed. ${ }^{13,14}$ Before entering the cavity, the light is spectrally filtered, yielding a

20 bandwidth of $400-800 \mathrm{~nm}$ ( $300 \mathrm{~mW}$ visible output power). This matches the bandwidth of the high reflectivity mirror coatings. At its intersection with the chip, the beam diameter is $c a .600 \mu \mathrm{m}$, which, with the per-pass optical pathlength $(l)$ through solution of $320 \mu \mathrm{m}$, yields a probed sample volume of $\sim 90 \mathrm{~nL}$.

25 Light exiting the cavity is fibre-coupled to a grating spectrograph (Andor Technology, SR-303i), which disperses its component wavelengths onto a CCD camera (Andor Technology, Newton). Spectra are acquired with a $3.66 \mathrm{~ms}$ acquisition time, at a repetition rate of $273 \mathrm{~Hz}$. The data rate is limited only by the 30 camera's acquisition settings / transfer rate. In other applications with more sophisticated cameras we have recorded full visible spectra with acquisition times of $10 \mu \mathrm{s}$ at $>600 \mathrm{~Hz} .^{13}$

In this proof-of-principle demonstration, we monitored droplets or 'slugs' of aqueous $\mathrm{K}_{2} \mathrm{IrCl}_{6}$ solution ( $\mathrm{Ir}(\mathrm{IV})$ ), during 35 their flow through a microfluidic channel in a toluene carrier phase. Before droplet-flow measurements, the detection sensitivity was determined in a series of single-wavelength continuous flow measurements. The absorption spectrum of Ir(IV), shown inset in Fig. 2, displays a maximum at $487 \mathrm{~nm}$. The

40 intensity, $I$, of $487 \mathrm{~nm}$ light transmitted through the cavity was recorded as solutions containing different concentrations of $\operatorname{Ir}(\mathrm{IV})$ were flowed continuously through the cell. The reference, $I_{0}$, was determined from measurements with pure solvent flowing through the chip. Fig. 2 shows $I_{0} / I-1$ as a function of [ $\left.\operatorname{Ir}(\mathrm{IV})\right]$. At
45 low concentrations, the plot displays a linear dependence on concentration (i.e., $I_{0} / I-1 \propto A$ ), with curvature at higher concentrations which may be attributed to a breakdown in the approximations leading to Equation 1. ${ }^{14,15}$ An error-weighted regression through the linear region provides a measure of the 50 limit of detection (LOD) of $1.4 \mu \mathrm{M}$ for $\operatorname{Ir}(\mathrm{IV})$, determined from the $3 \sigma$ uncertainty on the intercept. The minimum detectable perpass absorbance, $A_{\min }$, is $1.8 \times 10^{-4}$. The absorption coefficient, $\alpha$, is given by $\alpha=2.3026 \varepsilon C$, where the $C$ is the concentration and $\varepsilon$ the extinction coefficient, equal to $4000 \mathrm{M}^{-1} \mathrm{~cm}^{-1},{ }^{16}$ for $\operatorname{Ir}(\mathrm{IV})$ at ${ }_{55} 487 \mathrm{~nm}$. A limit of detection of $C_{\min }=1.4 \mu \mathrm{M}$ corresponds to a minimum detectable absorption, $\alpha_{\min }$, of $1.3 \times 10^{-2} \mathrm{~cm}^{-1}$.

To compare with single pass measurements, Equation (1) can be used to determine values for the CEF. At $487 \mathrm{~nm}$ the slope of the linear fit in Fig. 2 yields a CEF of 102, corresponding to an ${ }_{60}$ effective path length, $l_{\text {eff }}=\mathrm{CEF} \times l$, of $3.26 \mathrm{~cm}$. It is worth reiterating that this was achieved with a standard chip without any precision optical surfaces in just a $55 \mathrm{~ms}$ acquisition time. It is clear, even in these preliminary efforts, that the sensitivity of the BBCEAS technique surpasses that of previous absorption ${ }_{65}$ measurements in microfluidic chips (see, for example, Refs 1 and ${ }^{17}$ and references therein).

In the present work, the number of light passes through the cavity is primarily limited by the poor optical quality of the microfluidic chip itself. In previous liquid-phase cavity-based 70 studies, $\alpha_{\min }$ values in the range $10^{-3}-10^{-7} \mathrm{~cm}^{-1}$ have been achieved using high quality optical components (see Table II in Ref 18), indicating that by improving the optical quality of the chip alone, there is scope to improve the measurement sensitivity by several orders of magnitude.

75 In addition to the small volumes probed and the high sensitivity, a major advantage of this approach is its spectral bandwidth. To illustrate these combined attributes, Fig. 3 shows the absorption spectrum of $\sim 6 \mu \mathrm{L}$ 'slugs' of $40 \mu \mathrm{M}$ aqueous $\operatorname{Ir}(\mathrm{IV})$ solution flowing rapidly $(0.35 \mathrm{ml} / \mathrm{min})$ through the chip in 80 a toluene carrier phase. The temporal and wavelength-resolved spectra are shown in Fig. 3A. For these measurements, $I_{0}$ was the signal recorded in the presence of the carrier phase only. Fig. 3B highlights the excellent signal to noise achieved for an individual spectrum despite the short, $3.66 \mathrm{~ms}$, acquisition time. The time 85 resolution is demonstrated by the absorbance at $487 \mathrm{~nm}$ shown in Fig. 3C. Each individual spectrum contains a spectrally flat background component which we believe arises from scatter at the interface between the slug and a thin lubricating layer of carrier phase at the walls of the chip. As the absorption spectrum ${ }_{90}$ of $\operatorname{Ir}(\mathrm{IV})$ is known, this scattering contribution is easily quantified and subtracted (see Fig. 3B).

At both ends of the Ir(IV) slugs, lensing effects result in a large increase in the scattering. Only as the main body of the slug passes into the beam is the absorbance spectrum of $\operatorname{Ir}(\mathrm{IV})$ 95 recovered, (Fig. 3B). The large scattering-induced signals at the start and end of each droplet provide convenient signals for triggering acquisition during droplet flow, and could potentially be exploited for droplet shape analysis.

The signal to noise ratio achieved permits an alternative 100 estimate of $\alpha_{\min }$. From the $3 \sigma$ uncertainty in the baseline of the plot in Fig $3 \mathrm{C}, \alpha_{\min }(487 \mathrm{~nm})$ is estimated to be $9.5 \times 10^{-3} \mathrm{~cm}^{-1}$, close to the value obtained from the fit to the data in Fig. 2. 

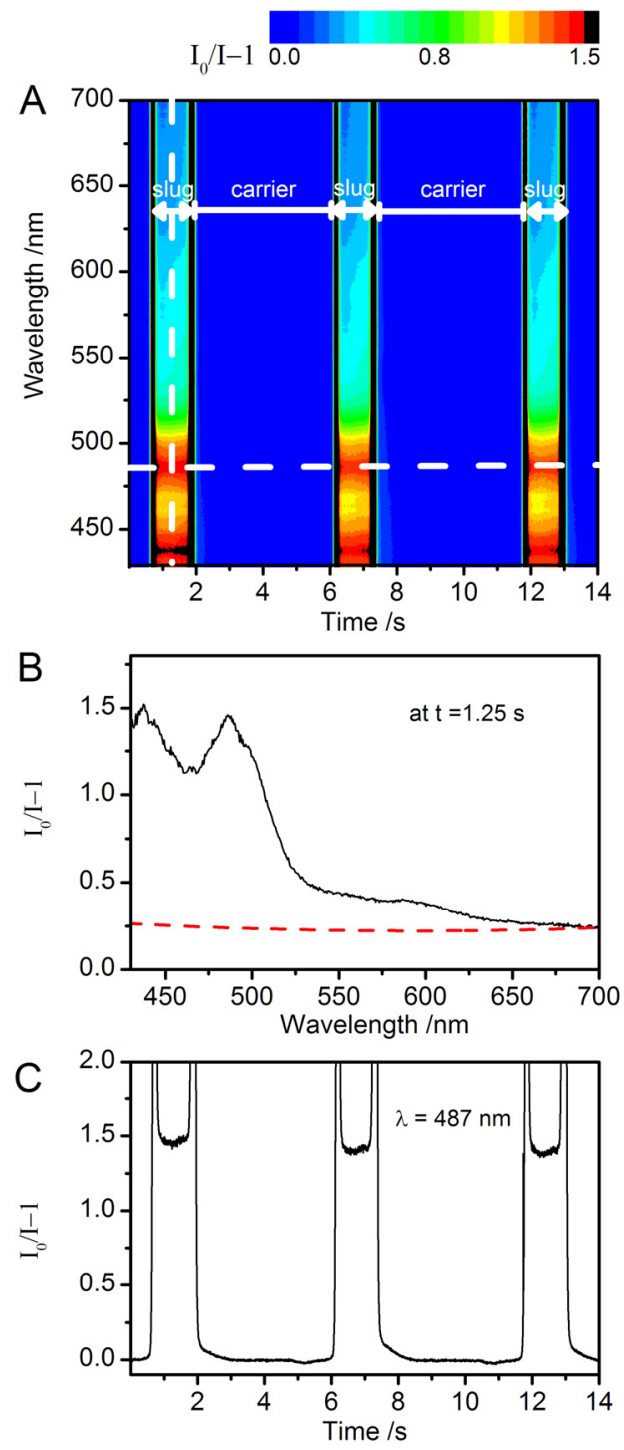

Fig. 3 (A) Contour plot of the BBCEAS-measured spectra as a function of time during a flow $\left(0.35 \mathrm{~mL} \mathrm{~min} \mathrm{~m}^{-1}\right)$ of $\operatorname{Ir}(\mathrm{IV})$ slugs through the microfluidic chip. (B) Spectral cut through the contour plot at $1.25 \mathrm{~s}$ (red dashed line represents a baseline scattering contribution) and (C) a temporal cut through at $487 \mathrm{~nm}$.

Given the proof-of-principle nature of this study, it is pertinent to discuss the future potential, and the limitations, of BBCEAS for microfluidic sensing. One of the key factors not already 10 discussed, which restricts the detection limit of the technique, is the stability of the supercontinuum source. Due to drift in the BBCEAS signal, increasing the signal integration time by a factor of 100 , to $0.366 \mathrm{~s}$, only results in a factor of two improvement in $\alpha_{\min }(487 \mathrm{~nm})$. The drift may be reduced by improving the chip and 15 cavity design, but a significant component is inherent in the SC source itself. To some extent this is the price paid for the intense, broadband coverage. Rapid switching between $I_{0}$ and $I$ may mitigate against this and would be well-suited to the study of rapidly flowing droplets.

20 As discussed above, the sensitivity of the technique is expected to improve markedly with minor modifications to the chip design (e.g., by incorporating optical quality surfaces). The large slug volumes used here could also be reduced substantially without losing sensitivity by using chip designs and dimensions that more 25 closely match the total droplet/slug volume with that probed by the cavity beam

A key advantage of the BBCEAS technique is that the broadband character of the absorption measurements allows the separation of contributions from species whose absorption spectra 30 overlap. This may be potentially useful for probing reactions within droplets, ${ }^{19}$ and, coupled with the fast temporal response, we envisage the technique being of considerable value in studying intra-droplet kinetics.

In summary, these results represent the first application of 35 broadband cavity-enhanced absorption spectroscopy to the analysis of microfluidic samples. The technique provides full visible broadband spectral measurement with both high detection sensitivity and excellent temporal and spatial resolution.

We would particularly like to thank Joanna Davies and Dr. João ${ }_{40}$ Cabral at Imperial College London for fabricating the chips used in this work. This work was funded by grants from EPSRC (EP/G027838/1), DARPA (QuBE: N66001-10-1-4061) and the EMF Biological Trust.

\section{Notes and references}

$4^{a}$ Department of Chemistry, University of Oxford, Physical and Theoretical Chemistry Laboratory, South Parks Road, Oxford OXI 3QZ, UK; e-mail stuart.mackenzie@chem.ox.ac.uk

${ }^{b}$ Department of Chemistry, University of Oxford, Chemistry Research Laboratory, 12 Mansfield Rd, Oxford OX1 3TA, UK; e-mail

501laire.vallance@chem.ox.ac.uk

1. B. Kuswandi, Nuriman, J. Huskens and W. Verboom, Anal. Chim. Acta, 2007, 601, 141-155.

2. K. B. Mogensen and J. P. Kutter, Electrophoresis, 2009, 30, S92$55 \mathrm{~S} 100$

3. P. J. Viskari and J. P. Landers, Electrophoresis, 2006, 27, 1797-1810.

4. M. L. Adams, M. Enzelberger, S. Quake and A. Scherer, Sens. Actuator A-Phys., 2003, 104, 25-31.

5. L. Malic and A. G. Kirk, Sens. Actuator A-Phys., 2007, 135, 515-524.

60 6. G. Berden and R. Engeln (Eds), Cavity Ring-Down Spectroscopy Techniques and Applications, Wiley, 2009.

7. C. Vallance, New J. Chem., 2005, 29, 867-874.

8. M. Schnippering, S. R. T. Neil, S. R. Mackenzie and P. R. Unwin, Chem. Soc. Rev., 2011, 40, 207-220.

65 9. H. Salimi-Moosavi, Y. T. Jiang, L. Lester, G. McKinnon and D. J. Harrison, Electrophoresis, 2000, 21, 1291-1299.

10. L. Billot, A. Plecis and Y. Chen, Microelectron. Eng., 2008, 85, 1269-1271.

11. G. Berden, R. Peeters and G. Meijer, International Reviews in Physical Chemistry, 2000, 19, 565-607.

12. C. Harrison, J. T. Cabral, C. M. Stafford, A. Karim and E. J. Amis, J. Micromech. Microeng., 2004, 14, 153-158.

13. L. van der Sneppen, G. Hancock, C. Kaminski, T. Laurila, S. R. Mackenzie, S. R. T. Neil, R. Peverall, G. A. D. Ritchie, M. Schnippering and P. R. Unwin, Analyst, 2010, 135, 133-139.

14. S. E. Fiedler, A. Hese and A. A. Ruth, Rev. Sci. Instrum., 2005, 76, 7.

15. S. E. Fiedler, A. Hese and A. A. Ruth, Rev. Sci. Instrum., 2005, 76, 1.

16. L. Moggi, G. Varani, M. F. Manfrin and V. Balzani, Inorg. Chim. Acta, 1970, 4, 335-341.

80 17. C. F. A. Floquet, V. J. Sieben, A. Milani, E. P. Joly, I. R. G. Ogilvie, H. Morgan and M. C. Mowlem, Talanta, 2011, 84, 235-239.

18. M. Islam, L. N. Seetohul and Z. Ali, Appl. Spectrosc., 2007, 61, 649658.

19. L. N. Seetohul, Z. Ali and M. Islam, Anal. Chem., 2009, 81, 4106854112 . 